\title{
Coding-Aware Peer-to-Peer Data Repair in Multi-Rate Wireless Networks - A Game Theoretic Analysis
}

\author{
Hsiao-Chen Lu, Student Member, IEEE, Wanjiun Liao, Fellow, IEEE, Meng Chang Chen, and Musaed A. \\ Alhussein
}

\begin{abstract}
Recent research shows that in wireless wide area networks (WWANs), users who subscribe to multicast traffic from WWAN can exchange network-coded packets with one another via their secondary radio interfaces such as $\mathrm{Wi}-\mathrm{Fi}$ in order to efficiently recover lost packets from the WWAN. Different from existing works which assume users are cooperative, in this work, we model the users as selfish players in the network-coding based peer-to-peer packet repairing game. To stimulate the users' cooperation, we introduce a payment-based incentive mechanism in the packet repairing game. The utility function of a user/player is also formulated to reflect both the number of useful packets and the available resource. Through analysis of the packet repairing game, we show that the optimal strategy for a user can be derived only with its local information. The impact of the pricing rules and the convergence conditions of the packet repairing game is also analyzed. We show theoretically as well as by simulation that under proper conditions, the packet repairing game can converge to the best case where each user can acquire all of its missing packets. Via computer simulations, we also show that with the proposed selection criteria, the packet repairing game is both effective and efficient: not only can the utilities of the players be greatly improved, but also the convergence time of the game and the utility gain of the players are comparable to those of the ideal case where every user is always willing to forward packets to others.
\end{abstract}

Index Terms-peer-to-peer data repair, network coding, multicast, game theory.

\section{INTRODUCTION}

$\mathbf{I}$ $\mathrm{N}$ the past few years, network coding (NC) was recognized as an efficient approach for information dissemination, widely applied to file sharing [1] and data storage [2]. In [3], network coding theory was further utilized to improve

Manuscript received April 15 2012; revised July 27 2012; accepted Oct 1 2012. The associate editor coordinating the review of this paper and approving it for publication was Prof. Sherman Shen.

This work was supported in part by the Excellent Research Projects of National Taiwan University, under Grant Number AE00-00-04, and in part by the National Science Council (NSC), Taiwan, under Grant Number NSC992221-E-002-029-MY3 and the Thematic Research Program, Academic Sinica, Taiwan.

H.-C. Lu is with the Graduate Institute of Communication Engineering, National Taiwan University, Taipei, Taiwan (e-mail: f95942045@ntu.edu.tw).

W. Liao (corresponding author) is with the Department of Electrical Engineering and the Graduate Institute of Communication Engineering, National Taiwan University, Taipei, Taiwan, and with Research Center for Information Technology Innovation, Academia Sinica, Taiwan (e-mail: wjliao@ntu.edu.tw)

Meng Chang Chen is with the Institute of Information Science, Academic Sinica, Taipei, Taiwan (e-mail: mcc@iis.sinica.edu.tw).

Musaed A. Alhussein is the Department of Computer Engineering, King Saud University, Kingdom of Saudi Arabia (e-mail: musaed@ ccis.ksu.edu.sa). the performance of multicasting. Specifically, with network coding, instead of forwarding native packets, intermediate forwarding nodes will linearly combine the received packets to generate a coded packet, and forward the coded packet to other nodes. The coefficients for linear combination, which form a coefficient vector, will be carried together with the coded packet. For any node, if the coefficient vectors of the received coded packets can form an invertible square matrix, the original native packets can be re-constructed. [4] is the first work that verifies the feasibility of network coding in wireless communications systems. By a practical test-bed implementation and study, it is demonstrated that utilizing the broadcast nature of the wireless medium, significant performance gain can be achieved by network coding. As a result, network coding has recently been applied widely in the field of wireless communications, achieving benefits including throughput maximization [4], energy efficiency [5] and privacy preservation [6].

In this paper, we focus on the network coding based peer-topeer multicast data repair problem, which can be described as follows. Suppose that users whose devices are equipped with both Wireless Wide Area Networks (WWANs) and Wireless Local Area Networks (WLANs) radio interfaces subscribe to receive multicast traffic from the WWAN. The WWAN base station (BS) will first broadcast a batch of multicast data packets to the subscribers. Due to wireless channel errors, a user may only receive a subset of the batch packets. Traditionally, the WWAN BS needs to re-transmit the missing packets for the users. However, when user devices are equipped with shortrange high-speed radio interfaces such as IEEE $802.11 \mathrm{Wi}$ $\mathrm{Fi}$, it is more efficient to let the users locally repair missing packets by distributing their received packets to one another through the short-range high-speed radio [7-9]. Moreover, network coding can be applied to enhance the performance of peer-to-peer data repair. For example, in Fig. 1, nodes A, B, $\mathrm{C}$, and D are the subscribers of a multicast flow and they have received different subsets of packets from the WWAN BS, who has sent packets $P_{1}, P_{2}, P_{3}, P_{4}$. In this example, nodes $\mathrm{A}$ and $\mathrm{C}$ have received all packets and they could help forward them to nodes $\mathrm{B}$ and $\mathrm{D}$, who have received only partial packets. In this example, we show that with network coding, only two transmissions are required for the users to cooperatively repair packets for one another, while four transmissions are required in the direct transmission case. Therefore, the time required for packet repairing can be significantly reduced with 


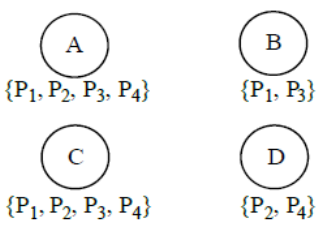

\begin{tabular}{|l|l|l|}
\hline & Transmission Schedule & $\begin{array}{l}\text { Single-rate } \\
\text { transmission count }\end{array}$ \\
\hline Direct transmission & $\begin{array}{l}\mathrm{A} \rightarrow \mathrm{B}: \mathrm{P}_{2}, \\
\mathrm{~A} \rightarrow \mathrm{B}: \mathrm{P}_{4}, \\
\mathrm{C} \rightarrow \mathrm{D}: \mathrm{P}_{1}, \\
\mathrm{C} \rightarrow \mathrm{D}: \mathrm{P}_{3} .\end{array}$ & 4 \\
\hline Network coding & $\begin{array}{l}\mathrm{A} \rightarrow \mathrm{B}, \mathrm{D}: \mathrm{P}_{1}+\mathrm{P}_{2}, \\
\mathrm{C} \rightarrow \mathrm{B}, \mathrm{D}: \mathrm{P}_{3}+\mathrm{P}_{4} .\end{array}$ & 2 \\
\hline
\end{tabular}

Fig. 1. An example demonstrating the efficiency of network coding in peerto-peer data repair.

network coding. The network-coding based cooperative peerto-peer data repair (or information exchange) problem has been studied in [7-9] under the single rate network model. The problem is proved to be NP-hard and some heuristic solutions have been proposed.

Previous studies on the cooperative NC-based data repair problem focus solely on the single transmission rate network model. For WLAN technologies such as IEEE 802.11, a transmitter can select different transmission rates according to the channel condition between the transmitter and the receiver. The studies in [10-13] show that the selection of transmission rates will not only impact the efficiency of network coding, but also lead to a tradeoff between network coding gain and transmission delay. In [11], we demonstrate that the solutions proposed in [7-9], which seek to minimize the number of re-transmissions, may lead to longer packet repairing delays in multi-rate scenarios. We then propose a Markov decision process-based dynamic programming solution for rate and forwarder selection in $\mathrm{NC}$-enabled multi-rate wireless relay networks. The transmission rate selection problem for NCenabled WLAN is studied in [12][13], and a centralized and a distributed solutions are proposed, respectively. However, in the previous works [7-13], the selfish nature of users is completely overlooked. In practice, a user, when forwarding its received packet to others, will not only consume its power but also have to give up the opportunity of receiving innovative packets from others. A packet is innovative to a receiving user if the coefficient vector associated with the packet does not fall in the vector space spanned by the coefficient vectors of the coded packets already received by the user. When a user receives an innovative packet from another user, its chance of decoding the original multicast batch packets is increased. If the number of innovative packets a user cumulatively received equals the size of the multicast batch, the original batch packets can be re-constructed. On the contrary, sharing packets with other users cannot instantly increase the chance of decoding packets. As a result, a selfish user naturally tends to be non-cooperative (i.e., not willing to share its received packets with others). In addition, since users are repairing packets for one another using wireless LAN technology which is rather decentralized, a user will only have local information regarding the status of its neighbors. Considering the multi-rate impact, the selfish nature of the users, and the de-centralized network scenario, a new model to characterize the behavior of users as well as a tailored solution to peer-to-peer data repair is indeed indispensable.

In this work, we propose a game-theoretic model to analyze the peer-to-peer data repair problem in NC-enabled multirate networks. As has been extensively applied to economics, game theory is also an ideal tool for analyzing the behavior of users in wireless networks [14]. We model the users as selfish players in a packet repairing game. Due to the lack of a centralized controller, each of the selfish users will make its own packet forwarding decision only to optimize its wellbeing. To stimulate the users' cooperation, we introduce a payment-based incentive mechanism to the packet repairing game. We then formulate the utility function for the users and show that the optimal strategies for a user can be derived based only on its local information. The pricing rules and the convergence conditions of the packet repairing game are also analyzed. We show theoretically as well as by simulation that under proper conditions, the packet repairing game can converge to the best case in which each user can acquire all of its missing packets. Moreover, via computer simulations, we demonstrate that with the proposed selection criteria, the packet repairing game is both effective and efficient: not only can the utilities of the players be greatly improved, but also the convergence time of the game and the utility gain of the players are comparable to those of the ideal case where each user is always willing to forward packets to others.

The rest of this paper is organized as follows. Our system model is presented in Section II. The proposed game-theoretic model for the NC-based peer-to-peer data repair problem is explained in Section III. In Section IV, the analyses of the packet repairing game, including the optimal strategies of the players, the pricing rule and the convergence conditions, are presented. We show and discuss the simulation results in Section V. Finally, Section VI concludes this paper.

\section{SYSTEM SCENARIO}

Consider that in a wireless wide area network (WWAN), there are user devices (referred to as users or nodes hereafter) who subscribe to the same multicast content. The WWAN base station (BS) is the multicast source, who broadcasts multicast packets in batches, as shown in Fig. 2. Let a batch of multicast traffic consist of $N$ fix-sized packets. Due to wireless channel errors, each subscriber may only receive a subset of the batch packets. At this time, the users who are equipped with wireless LAN (such as IEEE $802.11 \mathrm{Wi}-\mathrm{Fi}$ ) interfaces could repair missing packets for one another by re-transmitting coded packets (i.e., a linear combination of the native batch packets). We assume that each player can support transmission rates $r_{1}, r_{2}, \cdots, r_{C}$ for packet forwarding via WLAN, and without loss of generality, we assume $r_{1}<r_{2} \cdots<r_{C}$. 


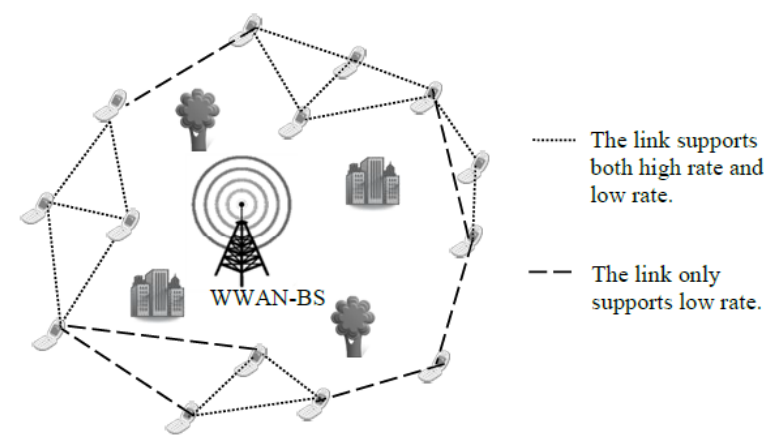

Fig. 2. An example for multi-rate WLAN peer-to-peer data repair.

Since each user has different distances and channel conditions to other users, a coded packet sent by a user's WLAN at a certain rate can only be successfully decoded by a subset of all the other users. We assume that each user can estimate the channel conditions to their neighbors by listening to the control messages (such as RTS or CTS) sent by the neighbors. In this way, a user is aware of which neighbors can decode the packet sent at each rate. For example, in Fig. 2, assume that each user supports two transmission rates. Then we use two different dotted lines to denote the rates supportability among the users. Generally, the better the channel quality, the higher the achievable transmission rate. For a user not equipped with Wi-Fi interfaces, it could only wait for the WWAN-BS to repair the missing batch packets, and thus will not participate in the peer-to-peer data repair process. In the following, we focus only on the data repair process among those users who are equipped with both WWAN and WLAN interfaces. For such users, the problem at hand is whether or not to share its own packets with others at each transmission opportunity. If a user is willing to share its packet with others, it will generate a coded packet, select an appropriate transmission rate to forward the packet, and then contend for transmission. For simplicity, we assume that the MAC technology is timeslotted, and a packet transmission is only allowed to start at the beginning of a time slot. At most one packet may be transmitted in one time slot, and the transmission of one packet may span multiple slots. We assume that when a user tries to forward a coded packet, it will follow a CSMA/CAlike contention mechanism to access the WLAN channel. We also assume that the contention is fair among all users, i.e., a contention of $K$ users results in a $1 / K$ success probability for each user.

Since each user has different native or coded multicast batch packets, to enable efficient packet repairing, the users need to exchange information regarding their received or missing packets, which may incur extra signaling overhead. To enable efficient exchange of packet information, in [15] the authors proposed the null-space vector feedback mechanism. The null space vector of a user is a vector randomly chosen from the null space of the coefficient vectors associated with the packets already received by the user. With the null-space feedback scheme, a user will piggyback its null-space vector whenever it transmits a packet. When one user receives a packet from another, it will extract and record the null-space vector of that user. Then a user can check if the coded packet it generates is innovative to another user in the following way. Let the coefficient vector of the coded packet generated by itself be $x_{i}$. Let the coefficient vectors of the packets already received by another user be denoted by $\left\{y_{1}, \ldots, y_{k}\right\}$ and the corresponding null-space vector by $n_{j}$. If the inner-product between $x_{i}$ and $n_{j}$ is not zero, $x_{i}$ cannot be spanned by $\left\{y_{1}, \ldots, y_{k}\right\}$ because any vector in the vector space spanned by $\left\{y_{1}, \ldots, y_{k}\right\}$ is orthogonal to $n_{j}$. Thus, the coded packet associated with $x_{i}$ is innovative to the user associated with $n_{j}$. In our system, we assume that each user supports the null-space vector feedback mechanism. Moreover, each user will estimate the channel conditions to its neighbors according to the signal strengths of the received packets or control signals from the neighbors. In this way, each time a user attempts to transmit a coded packet at a certain rate, it can estimate for which neighbors this packet will be innovative.

\section{A GAME-THEORETIC MODEL FOR THE NC-BASED PEER-TO-PEER DATA REPAIR PROBLEM}

In this section, we propose a game-theoretic model to analyze the behavior of users during the packet reparing process. We refer to the users as the players of the packet repairing game, and denote the number of players by $K$. The packet repairing game we define is essentially a repeated game - whenever players sense the WLAN channel idle, each of them will make a decision on which action to take. We assume that the action set of every user is the same, denoted by $A=\left\{a_{0}, a_{1}, \ldots, a_{C}\right\}$, where $a_{0}$ means refusing to forward a coded packet to others (and thus will not contend for transmission) and $a_{i}$ means forwarding a coded packet with transmission rate $r_{i}$. The strategy of the $i^{\text {th }}$ player, denoted by $s_{i}$, is defined as the action taken by the player. Hence, we have $s_{i} \in A, 1 \leq i \leq K$. The strategy profile of the game, denoted by $S$, is then the combination of the strategies of all the players, i.e., $S=\left(s_{1}, s_{2} \ldots, s_{K}\right)$.

Due to the selfish nature, when a user is playing the packet repairing game, it takes an action only to maximize its instant utility. Therefore, the formulation of the utility function greatly impacts the players' behavior, and it must properly reflect the well-being of a player under all possible strategy profiles. In the packet repairing game, a player's desire is to accumulate innovative packets as fast as possible in order to efficiently and effectively recover the original multicast batch packets. A player will not forward its packets to others because such action cannot enhance its own packet space and it will further consume power. Consequently, without extra incentive, peerto-peer data repair will never occur, which results in poor multicast efficiency and under-utilization of WLAN resource. To alleviate this problem, players who forward packets that are innovative to others should be rewarded. Accordingly, we introduce a payment mechanism in the packet repairing game: a player who successfully receives an innovative packet from a forwarder should pay money (either virtual or real money) to the forwarder. If the forwarded packet is not received by an 
intending receiver due to wireless channel errors, the receiver will not have to pay for the packet and thus the forwarder will not get the payment from the receiver. We assume that the price of a packet is determined by the rate at which it is transmitted, and the higher the transmission rate the higher the price, i.e., if we denote the price of the packet transmitted at rate $r_{i}$ as $\delta_{i}$, then we have $\delta_{1}<\delta_{2}<\cdots<\delta_{C}$. With the payment mechanism, the potential profit one player can earn from others becomes a motivation for packet forwardng.

Under the payment-based incentive mechanism, a rational user will always attempt to maximize its resource (in terms of both power and money) while also accumulating innovative packets as fast as possible. Therefore, we formulate the utility function of a player as the amount of resource weighted by the number of innovative packets it has received. To quantify the amount of resource a player owns, we convert the amount of money a user owns to an equivalent amount of power. Thus, let $\lambda$ be the amount of power that one unit of money can buy, then the total amount of resource a player owns is $(\lambda *$ money + power).

With the above definitions, we can now specifically formulate the utility function of a player. Let $n_{i}$ denote the number of innovative packets the $i^{t h}$ player has received, and $P_{i}$, the amount of resource the $i^{t h}$ player currently owns. Given a strategy profile $S=\left(s_{1}, s_{2} \ldots, s_{K}\right)$, we denote the strategy profile of players excluding the $i^{t h}$ player as $S_{-i}$. Then the utility function of the $i^{t h}$ player, which is a function of both $s_{i}$ and $S_{-i}$, is formulated as

$U_{i}\left(s_{i}, S_{-i}\right)=$ (amount of resource available at the $i^{\text {th }}$ player after playing strategy $\left.s_{i}\right) \times($ expected number of innovative packets the $i^{\text {th }}$ player has after playing strategy $s_{i}$ ).

If the $i^{t h}$ player decides to take action $a_{0}$ (i.e., refusing to forward), neither will it contend for channel access nor will it consume its power for packet forwarding. Thus, the utility function can be expressed by

$$
U_{i}\left(s_{i}=a_{0}, S_{-i}\right)=\left(P_{i}-\lambda \cdot C_{i}\left(S_{-i}\right)\right)\left(n_{i}+\mu_{i}\left(S_{-i}\right)\right),
$$

where $C_{i}\left(S_{-i}\right)$ denotes the expected payment the $i^{t h}$ player has to pay for receiving an innovative packet from others when it does not (or fails to) access the channel and other players play $S_{-i}$, and $\mu_{i}\left(S_{-i}\right)$ denotes the expected number of innovative packets the $i^{\text {th }}$ player can receive from others when it does not (or fails to) access the channel and other players play $S_{-i}$.

On the other hand, if the $i^{\text {th }}$ player decides to take action $a_{i}$, it will contend for transmission. Given $S_{-i}$, we denote the number of players (excluding the $i^{t h}$ player) attempting to access the channel by $N_{A}\left(S_{-i}\right)$. When the $i^{t h}$ player is contending for channel access, its success probability is $\frac{1}{N_{A}\left(S_{-i}\right)+1}$. If the $i^{\text {th }}$ player successfully accesses the channel, its utility will be $\left(\lambda \cdot G_{i}\left(s_{i}\right)+P_{i}-P_{t x}\right) n_{i}$, where $p_{t x}$ is the power consumption due to forwarding a packet and $G_{i}\left(s_{i}\right)$ is the expected payment the $i^{t h}$ player can earn from others by playing strategy $s_{i}$. Otherwise, if the $i^{t h}$ player fails to access the channel, which could happen with probability $1-\frac{1}{N_{A}\left(S_{-i}\right)+1}$, its utility will be the same as (1). Consequently, the utility function of the $i^{\text {th }}$ player taking forwarding action $a_{i}$ is an expected utility expressed as follows:

$$
\begin{gathered}
U_{i}\left(s_{i}=a_{i}, S_{-i}\right)=\frac{1}{N_{A}\left(S_{-i}\right)+1}\left(\lambda \cdot G_{i}\left(s_{i}\right)+P_{i}-P_{t x}\right) n_{i} \\
\quad+\left(1-\frac{1}{N_{A}\left(S_{-i}\right)+1}\right)\left(P_{i}-\lambda \cdot C_{i}\left(S_{-i}\right)\right)\left(n_{i}+\mu_{i}\left(S_{-i}\right)\right) .
\end{gathered}
$$

Whenever a player is not transmitting or receiving packets, it will keep sensing the channel. Once the channel is sensed idle, the player will first evaluate its utility under each action, and then take the action that maximizes its utility. If the taken action is not "refusing to forward," it will attemp to access the channel. After fair contention, one of the simultaneously contending players will win the channel and can forward a coded packet according to its strategy.

\section{ANALYSIS OF THE PEER-TO-PEER PACKET REPAIRING GAME}

\section{A. Optimal Strategy of a Player in the Packet Repairing Game}

According to (2), we have the following lemma.

Lemma 1: Each player has a dominant forwarding action. For the $i^{t h}$ player, $1 \leq i \leq K$, there exists $a_{f_{t}} \in A, a_{f_{t}} \neq$ $a_{0}$ such that $U_{i}\left(s_{i}=a_{f_{t}}, S_{-i}\right) \geq U_{i}\left(s_{i}=a_{j}, S_{-i}\right), \forall a_{j} \in$ $A, a_{j} \neq a_{0}$.

Proof: In (2), the selection of the forwarding strategy $s_{i}$ only affects the profit earned from others (i.e., $G_{i}\left(s_{i}\right)$ ), while all the other terms in (2) are completely independent of $s_{i}$. As a result, if we denote the action that maximizes $G_{i}\left(s_{i}\right)$ by $a_{f_{t}}$, i.e., $a_{f_{t}}=\arg \max G_{i}(a)$, then we have

$$
U_{i}\left(s_{i}=a_{f_{t}}, S_{-i}\right) \geq U_{i}\left(s_{i}=a_{j}, S_{-i}\right), \forall a_{j} \in A, a_{j} \neq a_{0} .
$$

Since for the $i^{\text {th }}$ player, taking action $a_{f_{t}}$ leads to no lower utility compared with other forwarding actions, a rational player will only consider either taking action $a_{f_{t}}$ or refusing to forward, depending on which of the two actions leads to higher utility. Thus, the optimal strategy of the $i^{t h}$ player can be derived according to (3):

$$
U_{i}\left(s_{i}=a_{f_{t}}, S_{-i}\right) \geq U_{i}\left(s_{i}=a_{0}, S_{-i}\right) .
$$

In other words, a user should forward with $a_{f_{t}}$ if (3) holds. Otherwise, it should refuse to forward.

Substituting (1) and (2) into (3), and after further simplification, we get the following:

$\left[\lambda \cdot G_{i}\left(a_{f_{t}}\right)+P_{i}-P_{t_{x}}\right] n_{i} \geq\left[P_{i}-\lambda \cdot C_{i}\left(S_{-i}\right)\right]\left(n_{i}+\mu_{i}\left(S_{-i}\right)\right)$.

If (4) holds, forward with $a_{f_{t}}$; otherwise, refuse to forward.

To evaluate whether or not (4) holds, we observe that the left-hand-side (LHS) of (4) is only affected by the dominant forwarding action of the $i^{\text {th }}$ player, while the right-handside (RHS) is mainly affected by the strategy profile of other players (i.e., $S_{-i}$ ). Since we consider a de-centralized peerto-peer packet repair scenario, when a player attempts to play a strategy, it only has local information such as the number of innovative packets received (i.e., $n_{i}$ ), its amount of resource (i.e., $P_{i}$ ), and the transmission power $p_{t x}$. To estimated $G_{i}\left(a_{f_{t}}\right)$, the expected payment the $i_{t h}$ player can earn from 
others by playing strategy $a_{f_{t}}$. Thus, the player should first check how many neighbors can successfully receive the packet forwarded by the rate associated with $a_{f_{t}}$, and then verify if the coded packet generated by itself is innovative to these neighbors by taking innner-products between the generated coded vector and the null-space vectors of these neighbors. By estimating to how many neighbors is the generated packet innovative and then multiplies the number by the price of a packet forwarded with action $a_{f_{t}}$ and the probability of success packet reception, $G_{i}\left(a_{f_{t}}\right)$ can be estimated (assuming that the packet reception event of each user is independent). Since the tranmission power and available resource are known to each player, the LHS of (4) can be estimated only by a player's local information. On the contrary, a player cannot calculate the RHS without the knowledge of other players' strategy profile. Nevertheless, the upper and lower bounds of $C_{i}\left(S_{-i}\right)$ and $\mu_{i}\left(S_{-i}\right)$ can be utilized to derived the upper and lower bounds of the RHS of (4). Since $C_{i}\left(S_{-i}\right)$ is the expected payment the $i^{t h}$ player has to pay out when it does not (or fails to) access the channel and other players play $S_{-i}$, it should be a convex combination of $\left\{0, \delta_{1}, \ldots, \delta_{C}\right\}$ and should be bounded between 0 and $\delta_{C} \cdot \mu_{i}\left(S_{-i}\right)$, the expected number of innovative packets the $i^{\text {th }}$ player can receive from others when it does not (or fails to) access the channel and other players play $S_{-i}$, should be bounded between 0 and 1 . As a result, the RHS of (4) is also bounded as follows:

$\left[p_{i}-\lambda \cdot \delta_{C}\right] n_{i} \leq\left[p_{i}-\lambda \cdot C_{i}\left(S_{-i}\right)\right]\left(n_{i}+\mu_{i}\left(S_{-i}\right)\right) \leq P_{i}\left(n_{i}+1\right)$.

When $\left[\lambda \cdot G_{i}\left(a_{f_{t}}\right)+P_{i}-P_{t_{x}}\right] n_{i}<\left(p_{i}-\lambda \cdot \delta_{C}\right) n_{i}$, or equivalently, $\lambda \cdot G_{i}\left(a_{f_{t}}\right)<p_{t x}-\lambda \cdot \delta_{C}$, the LHS of the inequality in (4) is absolutely smaller than the RHS, meaning that refusing to forward leads to absolutely higher utility. Similarly, if $\left[\lambda \cdot G_{i}\left(a_{f_{t}}\right)+P_{i}-P_{t_{x}}\right] n_{i}>P_{i}\left(n_{i}+1\right)$, the LHS of the inequality in (4) is absolutely greater than the RHS, meaning that forwarding with $a_{f_{t}}$ leads to absolutely higher utility. In these two cases, a player can locally derive its optimal strategy. However, if the LHS of (4) falls between the bounds of the RHS of (4), a player could not determine which one of $a_{0}$ and $a_{f_{t}}$ leads to higher utility unless other information regarding $S_{-i}$ is available. In such a case, we assume that a player will simply randomly choose one of $a_{0}$ and $a_{f_{t}}$ as its strategy.

For players that have already received $N$ innovative packets, no more coded packet of the same multicast batch can be innovative, which means $C_{i}\left(S_{-i}\right)=\mu\left(S_{-i}\right)=0$. Thus, the RHS of (4) becomes $P_{i} n_{i}$. As a result, the strategy selection criterion becomes: if $\left[\lambda \cdot G_{i}\left(a_{f_{t}}\right)+P_{i}-P_{t_{x}}\right] n_{i} \geq P_{i} n_{i}$, the player should forward with action $a_{f_{t}}$. Otherwise it should refuse to forward. After further simplification, it becomes: if $\lambda \cdot G_{i}\left(a_{f_{t}}\right) \geq P_{t_{x}}$, the player should forward with action $a_{f_{t}}$. Otherwise it should refuse to forward.

From the above inferences, we can summarize the optimal strategy of a player in the packet repairing game. Let $i$ be the index of the player:

1)When the player has not received $N$ innovative packets yet (i.e., $\left.n_{i}<N\right)$ : if $\lambda \cdot G_{i}\left(a_{f_{t}}\right)<P_{t_{x}}-\lambda \cdot \delta_{C}$, its optimal strategy is refusing to forward. If $\left[\lambda \cdot G_{i}\left(a_{f_{t}}\right)+P_{i}-P_{t_{x}}\right] n_{i}>$
$P_{i}\left(n_{i}+1\right)$, then its optimal strategy is taking action $a_{f_{t}}$.

2)When the player has received $N$ innovative packets: if $\lambda \cdot G_{i}\left(a_{f_{t}}\right) \geq p_{t_{x}}$, its optimal stratgy is to take action $a_{f_{t}}$. Otherwise, its optimal strategy is refusing to forward.

\section{B. Pricing Rules}

From the analysis of a player's optimal strategy, we can observe that the price of an innovative packet determines the expected payment a player can earn from forwarding a coded packet and greatly impacts a player's decision on forwarding. If the price of an innovative packet is too high, to avoid the high payment from receiving an innovative packet, a player will intentionally contend for transmission just to reduce other players' transmission probability even though its coded packet is not innovative to others. Once such a player successfully accesses the channel, no one can benefit from the transmitted packet and the wireless resource is wasted. To avoid such situations, the price of packets should be properly settled such that any player whose coded packet is innovative to no one will have no motivation to take any forwarding action. To achieve this, when $G_{i}\left(a_{f_{t}}\right)$ is zero, the LHS of (4) should always be smaller than the RHS of (4). Therefore,

$$
\left[P_{i}-P_{t_{x}}\right] n_{i}<\left[P_{i}-\lambda \cdot C_{i}\left(S_{-i}\right)\right]\left(n_{i}+\mu\left(S_{-i}\right)\right)
$$

should always hold under the given $\lambda, P_{t_{x}}$ and all possible $P_{i}, C_{i}\left(S_{-i}\right)$ and $\mu_{i}\left(S_{-i}\right)$. Note that when $n_{i}$ is zero, a player cannot forward any coded packet because it has not received any packet yet. When $\mu_{i}\left(S_{-i}\right)$ is zero, meaning that among the players excluding the $i^{t h}$ one, either no one is taking forwarding action or none of their coded packets is innovative to the $i^{\text {th }}$ player, $C_{i}\left(S_{-i}\right)$ will also be zero. In this case, (6) will also hold. When $n_{i}$ and $\mu_{i}\left(S_{-i}\right)$ are both nonzero, rearranging (6), we will get

$$
\lambda \cdot C_{i}\left(S_{-i}\right)<\frac{1}{n_{i}+\mu\left(S_{-i}\right)}\left(\mu\left(S_{-i}\right) P_{i}+n_{i} P_{t x}\right) .
$$

Note that the RHS of (7) is a convex combination of $P_{i}$ and $p_{t x}$ with nonzero conefficients because both $n_{i}$ and $\mu_{i}\left(S_{-i}\right)$ are nonzero. For each player who can possibly take forwarding actions, its resource $P_{i}$ must be greater than the transmission power $p_{t x}$. As a result, the RHS of (7) is smaller than $P_{i}$ and greater than $p_{t x}$. Since $C_{i}\left(S_{-i}\right)$ is a random variable bounded between 0 and $\delta_{C}$, to assure (7) under all possible nonzero $n_{i}$ and $\mu_{i}\left(S_{-i}\right)$, we must have $\lambda \cdot \delta_{C} \leq p_{t x}$.

Proposition 1: For efficient resource utilization, the proper pricing rule for the packet forwarding game is that the equivalent power of the price of an innovative packet forwarded with the highest rate $(\lambda \delta C)$ must be no larger than the transmission power of a coded packet (i.e., $p_{t x}$ ), i.e., $\lambda \cdot \delta_{C} \leq p_{t x}$.

\section{Convergence Conditions of the Packet Repairing Game}

When the optimal strategies of all players are refusing to forward, the status of every player will not change, making each of them take the same refusal action in the next round. Hence, the game will converge (or terminate) from the moment everyone takes the action of refusing to forward. The ideal termination condition is that all players have received $N$ 
innovative packets, and therefore no more packet transmissions are innovative to anyone, i.e., $G_{i}\left(a_{f_{t}}\right)=0, \forall i \in\{1, \cdots, K\}$. As discussed in Sec. IV-A, a player who has received $N$ innovative packets (referred to as a completed player hereafter) would take the dominant forwarding action only when $\lambda \cdot G_{i}\left(a_{f_{t}}\right) \geq p_{t x}$, which will hold for no player in this case. Hence, every player will refuse to forward and the game terminates. Since every player has received enough coded packets to recover the original multicast batch packets, we call a game terminated in this way a successful game.

However, it is also possible that there are some players who have not received $N$ innovative packets yet, but all players' optimal strategies are refusing to forward and the game terminates. We call such a case a premature convergence. According to Sec. IV-A, once a player who has not received $N$ innovative packet yet (referred to as a uncompleted player hereafter) satisfies $\lambda \cdot G_{i}\left(a_{f_{t}}\right) \leq p_{t x}-\lambda \cdot \delta_{C}$ or a completed player satisfies $\lambda \cdot G_{i}\left(a_{f_{t}}\right) \leq p_{t x}$, they will stop forwarding. Since the expected payment that a player can earn from forwarding a packet is non-increasing with time because other player's packet space is growing with time, once a player chooses to stop forwarding, it will always remain refusing to forward. To avoid pre-mature convergence, we observe that in $\lambda \cdot G_{i}\left(a_{f_{t}}\right) \leq p_{t x}-\lambda \cdot \delta_{C}$, the RHS is non-negative because of our pricing rule $\lambda \cdot \delta_{C} \leq p_{t x}$. If there is a nonzero gap between $\lambda \cdot \delta_{C}$ and $p_{t x}$, a player could denying forwarding even though its packet is still innovative to others (i.e., $G_{i}\left(a_{f_{t}}\right)$ is still positive). To stimulate packet forwarding and avoid premature convergence, we must have $\lambda \cdot \delta_{C}=p_{t x}$, such that a uncompleted player stops forwarding only when $\lambda \cdot G_{i}\left(a_{f_{t}}\right) \leq p_{t x}-\lambda \cdot \delta_{C}=0$, i.e., when $G_{i}\left(a_{f_{t}}\right)=0$.

Proposition 2: The highest price of a coded packet, $\delta_{C}$, should satisfy $\lambda \cdot \delta_{C}=P_{t x}$. In this way, an uncompleted player will stop forwarding if and only if its coded packet is innovative to no one.

Theorem 1: If Proposition 2 holds, the peer-to-peer packet repairing game will converge successfully when the following two conditions are satisfied: 1) when the WWAN-BS is broadcasting the multicast batch packets, each of the packets is successfully received by at least one player (or equivalently, the collective packet space of the players has dimension $N$ ), and 2) the high-rate topology graph of the players, constructed by taking each player as a vertex and creating edges between each player (vertex) and other players (vertices) it can reach by $r_{C}$, is a connected graph.

Proof: First, we can prove that before the game terminates, at least one player must have received $N$ innovative packets. If not, this means all the players refuse to forward when all of them have received less than $N$ innovative packets, and their packets must span the same packet space; otherwise one player can generate innovative packets to its neighbors, which is a non-empty set of players because of topology connectivity, and according to Proposition 2, the player will not refuse to forward. Hence, the dimension of the collective packet space of the players is smaller than $N$, which contradicts with the assumption that each multicast batch packet is successfully received by at least one player.

Secondly, we prove that once a player has received $N$ innovative packets, before the game terminates, all the players will receive $N$ innovative packets. For a completed player, if there exists at least one uncompleted neighbor in its high rate neighborhood, it can always generate an innovative packet for the neighbors and send it with rate $r_{C}$, making an expected payment gain of at least $\delta_{C}$ (i.e., $G_{i}\left(a_{f_{t}}\right) \geq \delta_{C}$ ). Since $\lambda \cdot \delta_{C}=p_{t x}$ according to Proposition 2, for a completed player, as long as there exists at least one neighbor in its high rate neighborhood, $\lambda \cdot G_{i}\left(a_{f_{t}}\right) \geq \lambda \cdot \delta_{C}=p_{t x}$ will hold, which means it will not refuse to forward until all its neighbors in the high-rate neighborhood are completed. In this way, if one player has completely received $N$ innovative packets before the game terminates and the high-rate topology graph of the players is a connected graph, eventually all the players will completely receive $N$ innovative packets before the game terminates.

Since at least one player must have received $N$ innovative packets before the game terminates, and once a player has received $N$ innovative packets all the players will receive $N$ innovative packets before the game terminates, the peer-to-peer packet repairing game will converge successfully.

\section{PERFORMANCE EVALUATION AND DISCUSSIONS}

In this section, we discuss our computer simulations and the major results. The simulation environment is described as follows. We consider a $120 \mathrm{~m}$ by $120 \mathrm{~m}$ geographic area, where a WWAN BS is located at the center and a variable number of users are randomly distributed. All users are equipped with both WWAN and WLAN interfaces. The WWAN-BS will first broadcast a batch of 8 multicast packets, and each packet can be successfully received by each user with probability 0.7 . After the WWAN BS broadcasts the entire set of multicast batch packets, users will start peer-to-peer packet repairing using the IEEE 802.11b WLAN interfaces. Each user can support two transmission rates, namely, 5.5 Mbps and 11 Mbps. We also simulate the case where users can only support the low transmission rate (i.e., 5.5 Mbps) to evaluate the impact of rate supportability on the packet repairing game. To test the validity of Theorem 1, only when each multicast batch packet is successfully decoded by at least one user and the topology of users forms a connected graph will the peerto-peer packet repairing process be further simulated. Initially, we assume that each user has the same power level, which is equal to 200 units. The transmission power of a packet, $p_{t x}$, is set to 15 units and the amount of power that one unit of money can buy, $\lambda$, is set to 1.5. When users support multiple rates (i.e., both $11 \mathrm{Mbps}$ and $5.5 \mathrm{Mbps}$ ), according to Proposition 2 , the price of a packet forwarded at $11 \mathrm{Mbps}$ is $15 / 1.5=$ 10 units. Assuming that the packet price is a linear function of its transmission rate, the price of a packet transmitted at $5.5 \mathrm{Mbps}$ is then 5 units. If the users only support single rate (i.e., 5.5 Mbps), the price of a packet forwarded at 5.5 Mbps is again $15 / 1.5=10$ units. The time required for a packet transmission at $5.5 \mathrm{Mbps}$ and $11 \mathrm{Mbps}$ is 8 timeslots and 4 timeslots, respectively.

In the following, we show the simulation results on the average convergence time of the game and the average utility 


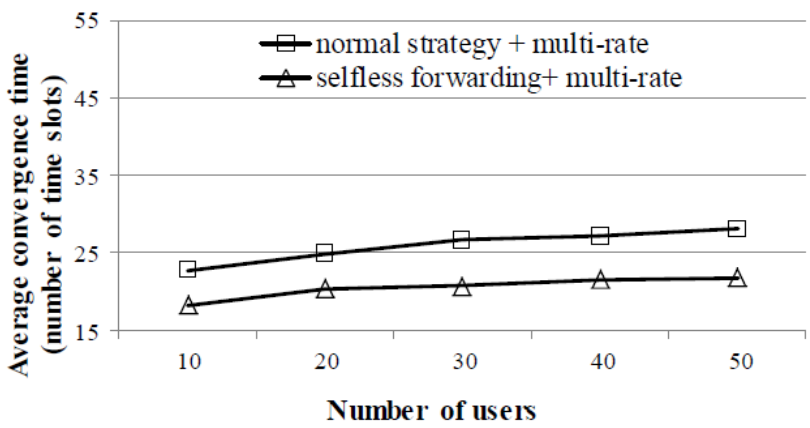

Fig. 3. The average convergence time of the packet repairing game under multi-rate scenarios

gain of the users, under different number of users. For each number of users, 300 random topologies are generated and the results are averaged. Aside from each user playing the game following the strategies proposed in Sec. IV-A (referred to as "normal strategies" in the following), for comparison, we also simulate an ideal situation where each user is not concerned with its available resource and is always willing to forward a coded packet to others (referred to as "selfless forwarding" in the following).

In all our simulations of both scenarios under both user capabilities, when the packet repairing game converges, all the users indeed have successfully received 8 innovative packets, which proves the validity of Theorem 1. In Fig. 3 and Fig. 4 , we show the average convergence time (in number of time slots) of the packet repairing game for multi-rate and singlerate scenarios, respectively, under different user strategies. Comparing the results in the two figures, we discover that with multi-rate support, the convergence time of the packet repairing game is shorter, which means better packet repairing efficiency. In both multi-rate and single-rate scenarios, the convergence time of the games with selfless forwarding is shorter than that with the normal strategies, showing that the selfish nature of users is indeed disadvantageous for packet repairing efficiency. However, the performance gaps between the normal strategies we derive and the selfless forwarding strategy are quite small. In the multi-rate scenario, the convergence time of normal strategies is only around $26 \%$ higher than that of selfless forwarding and the ratio is quite static with respect to the growing number of users. In the singlerate scenario, the gaps are even smaller, i.e., the convergence time of normal strategies is only around $8 \%$ higher than that of selfless forwarding. The results indicate that with the strategies we derive, the efficiency of the packet repairing game is comparable to that of the ideal situation where every user is always willing to forward packets.

In Fig. 5 and Fig. 6, we show the results of the average utility gain, defined as the average utility of users after the game converges divided by the average utility of users before the game starts, for multi-rate and single-rate scenarios, respectively, under two different user strategies. For both scenarios, the results show that all the average utilities are improved by around $40 \%$ after the game converges. In other

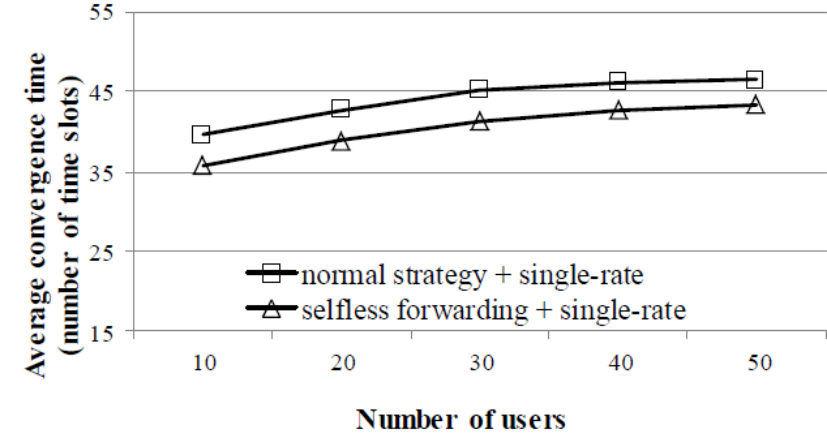

Fig. 4. The average convergence time of the packet repairing game under multi-rate scenarios

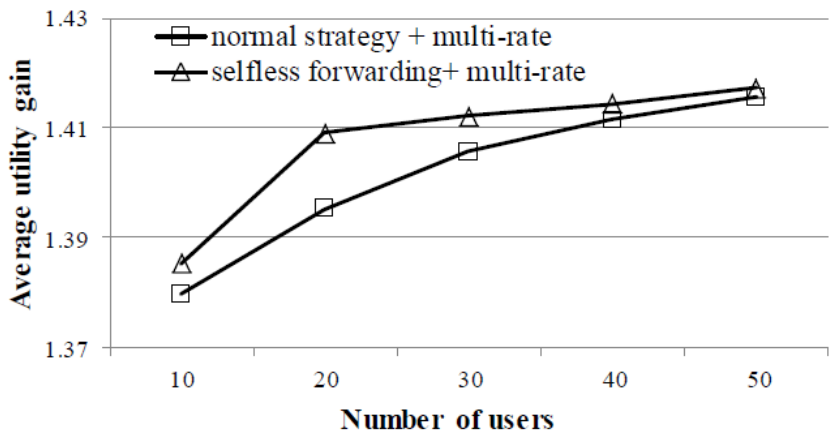

Fig. 5. The average utility gain of the packet repairing game under multi-rate scenarios

words, by playing the packet repairing game, users not only acquire their missing packets but also improve their utilities. Still, the average utility gains of our derived normal strategies and selfless forwarding are very close. Generally, the selfless forwarding strategy slightly outperforms the normal forwarding strategy; however, the gap diminishes as the number of users grows. The possible reason is that when the number of users grows, each user will have more neighbors, making a user more likely to forward a packet because the expected payment received from forwarding this packet is higher. As a result, when the number of users increases, the tendency of each user to forward packets is also higher, making the behavior of users as well as the game performance closer to those of selfless forwarding. Overall, the simulation results show that with the optimal strategies we derive for the packet forwarding game, not only can users make their forwarding decisions by locally available information in a very simple and efficient way, but also the performance of the game in terms of convergence time and average utility gain is quite close to those of the ideal (i.e., users are selfless) situation. 


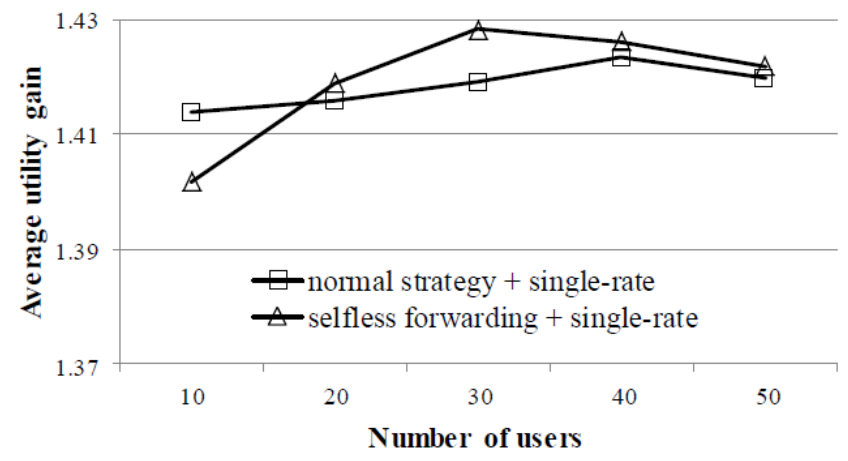

Fig. 6. The average utility gain of the packet repairing game under single-rate scenarios

\section{CONCLUSION}

In this paper, we analyze the network coding-based peer-topeer packet repairing problem under the assumption that users are selfish and they make decisions of packet forwarding only with local information and only to optimize their individual well-being. Under such circumstances, it is natural to apply a game-theoretic model to analyze the user behavior. We have constructed a game-theoretic model with a payment-based incentive mechanism for the network-coding based peer-to-peer packet repairing process, deriving the optimal strategies for the players with local information, and also finding the proper pricing rules in order to achieve efficient resource utilization and ideal convergence of the game. The simulation results show that with the proposed strategy, the packet repairing game is both effective and efficient; not only can the utilities of the players be greatly improved, but also the convergence time of the game and the utility gain of the players are comparable to those of the ideal case in which each user is always willing to forward packets to others.

\section{REFERENCES}

[1] C. Gkantsidis and P. Rodriguez, "Network coding for large scale content distribution," in Proc. IEEE INFOCOM' 05, Mar. 2005.

[2] A. Dimakis, P. Godfrey, Y. Wu, M. Wainwright, and K. Ramchandran, "Network coding for distributed storage systems," IEEE Transactions on Information Theory, vol. 56, no. 9, pp. 4539-4551, Sep. 2010.

[3] Z. Li, B. Li, and L. Lau, "On achieving maximum multicast throughput in undirected networks," IEEE Transactions on Information Theory, vol. 52, no. 6, pp. 24672485, Jun. 2006.

[4] S. Katti, H. Rahul, W. Hu, D. Katabi, M. Médard, and J. Crowcroft, "XORs in the air: practical wireless network coding," in Proc. ACM SIGCOMM' 06, Aug. 2006.

[5] T. Cui, L. Chen, and T. Ho, "Energy efficient opportunistic network coding for wireless networks," in Proc. IEEE INFOCOM' 08, Apr. 2008.
[6] P. Zhang, Y. Jiang, C. Lin, Y. Fan, and X. Shen, "Pcoding: Secure network coding against eavesdropping attacks," in Proc. IEEE INFOCOM' 10, Mar. 2010.

[7] X. Liu, S. Raza, C.-N. Chuah, and G. Cheung, "Network coding based cooperative peer-to-peer repair in wireless ad-hoc networks," in Proc. IEEE ICC' 08, May 2008.

[8] X. Liu, G. Cheung, and C.-N. Chuah, "Structured network coding and cooperative wireless ad-hoc peer-topeer repair for wwan video broadcast," IEEE Transactions on Multimedia, vol. 11, no. 4, pp. 730-741, June 2009.

[9] Y. Fan, Y. Jiang, H. Zhu, and X. Shen, "Pie: cooperative peer-to-peer information exchange in network coding enabled wireless networks," IEEE Transactions on Wireless Communications, vol. 9, no. 3, pp. 945-950, Mar. 2010.

[10] L. F. M. Vieira, A. Misra, and M. Gerla, "Performance of network-coding in multi-rate wireless environments for multicast applications," in Proc. IEEE MILCOM' 07, Oct. 2007.

[11] H.-C. Lu and W. Liao, "Cooperative multicasting in network-coding enabled multi-rate wireless relay networks," in Proc. IEEE INFOCOM' 12, Mar. 2012.

[12] T.-S. Kim, S. Vural, I. Broustis, D. Syrivelis, S. Krishnamurthy, and T. La Porta, "A framework for joint network coding and transmission rate control in wireless networks," in Proc. IEEE INFOCOM' 10, Mar. 2010.

[13] R. Kumar, S. Tati, F. de Mello, S. Krishnamurthy, and T. La Porta, "Network coding aware rate selection in multi-rate ieee 802.11," in Proc. IEEE International Conference on Network Protocols(ICNP)' 10, Oct. 2010.

[14] M. Felegyhazi and J. P. Hubaux, "Game theory in wireless networks: a tutorial," EPFL technical report, LCAREPORT-2006-002, Feb. 2006.

[15] D. Koutsonikolas, C.-C. Wang, and Y. Hu, "Ccack: Efficient network coding based opportunistic routing through cumulative coded acknowledgments," in Proc. IEEE INFOCOM’ 10, Mar. 2010.

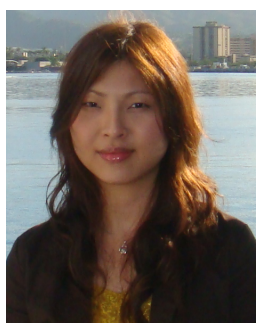

Hsiao-Chen Lu Hsiao-Chen Lu received her BS degree in Electrical Engineering from National Taiwan University (NTU), Taipei, Taiwan. She is currently a $\mathrm{PhD}$ candidate in the Graduate Institute of Communication Engineering, NTU. Her research interests are in the field of network planning and performance optimization for relay-assisted wireless access networks, especially for the IEEE 802.16j WiMAX network and the 3GPP Long Term Evaluation (LTE) network. She is a student member of IEEE. 


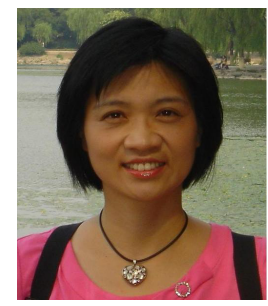

Wanjiun Liao Wanjiun Liao received her Ph.D. degree in Electrical Engineering from the University of Southern California, USA, in 1997. She is a Distinguished Professor of Electrical Engineering, National Taiwan University (NTU), Taipei, Taiwan, and an Adjunct Research Fellow of Research Center for Information Technology Innovation, Academia Sinica, Taiwan. Her research focuses on the design and analysis of wireless multimedia networking, cloud datacenter networking and green communications. Prof. Liao was on the editorial boards of IEEE Transactions on Wireless Communications and IEEE Transactions on Multimedia. She has also served on the organizing committees of many international conferences, including serving as Tutorial Co-Chair of IEEE INFOCOM 2004, TPC Vice Chair of IEEE GLOBECOM 2005 Symposium on Autonomous Networks, TPC Co-Chair of IEEE GLOBECOM 2007 General Symposium, TPC Co-Chair of IEEE VTC 2010 Spring and TPC Co-Chair of IEEE ICC 2010 Next Generation Networking and Internet Symposium. The papers she co-authored with her students won the Best Paper Awards of IEEE ICME 2000 and IEEE GLOBECOM 2011, and IEEE ComSoc 2011 Multimedia Communications Best Paper Award. She was a recipient of the Republic of China (R.O.C.) Distinguished Women Medal in 2000 and was elected as a Distinguished Lecturer of IEEE Communications Society (20112012). She is a Fellow of IEEE.

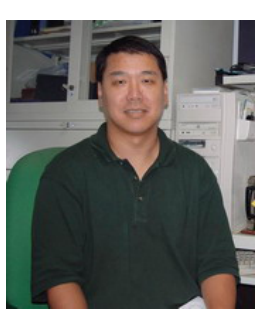

Meng Chang Chen Meng Chang Chen received the BS and MS degrees in Computer Science from National Chiao-Tung University, Taiwan, and the $\mathrm{PhD}$ degree in Computer Science from the University of California, Los Angeles, in 1989. He was with AT\& T Bell Labs from 1989 to 1992 . He then joined Institute of Information Science, Academia Sinica, Taiwan. From 1999 to 2002 and from 2008 to 2010, he served as Deputy Director of the institute. From 2000 to 2003, he served as the chair of Standards and Technology Transfer group of the National Science $\&$ Technology Program for Telecommunications Office (NTPO). His current research interests include wireless networks, information retrieval, and data and knowledge engineering.

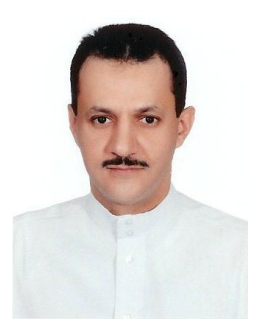

Dr. Musaed A. Al-Hussein Dr. Musaed A. AlHussein was born in Riyadh, Saudi Arabia. He received his B.S. degree in Computer Engineering from King Saud University, Riyadh, in 1988, and the M.S. and Ph.D. degrees in Computer Science and Engineering from University of South Florida, Tampa, Florida, in 1992 and 1997, respectively. Since 1997, he has been on the Faculty of the Computer Engineering Department, College of Computer and Information Science, King Saud University. His current research interests include wireless sensor networks and wireless ad hoc networking. 